\title{
Flutter, Postflutter, and Control of a Supersonic Wing Section
}

P. Marzocca, L. Librescu, W. A. Silva

Reprinted from

\section{Journal of Guidance, Control, and Dynamics}

Volume 25, Number 5, Pages 962-970

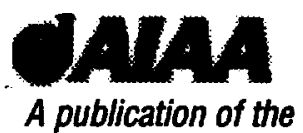

American Institute of Aeronautics and Astronautics, Inc.

1801 Alexander Bell Drive, Suite 500

Reston, VA 20191-4344 


\title{
Flutter, Postflutter, and Control of a Supersonic Wing Section
}

\author{
Piergiovanni Marzocca* and Liviu Librescu ${ }^{\dagger}$ \\ Virginia Polytechnic Institute and State University, Blacksburg, Virginia 24061-0219 \\ and \\ Walter A. Silva ${ }^{\ddagger}$ \\ NASA Langley Research Center, Hampton, Virginia 23681-2199
}

\begin{abstract}
A number of issues related to the flutter and postflutter of two-dimensional supersonic lifting surfaces are addressed. Among them there are the 1) investigation of the implications of the nonlinear unsteady aerodynamics and structural nonlinearities on the stable/unstable character of the limit cycle and 2) study of the implications of the incorporation of a control capability on both the flutter boundary and the postflutter behavior. To this end, a powerful methodology based on the Lyapunov first quantity is implemented. Such a treatment of the problem enables one to get a better understanding of the varfous factors involved in the nonlinear aeroelastic problem, including the stable and unstable limit cycle. In addidion, it constitutes a first step toward a more general investigation of nonlinear aeroelastic phenomena of three-dimensional lifting surfaces.
\end{abstract}

\begin{tabular}{|c|c|}
\hline & Nomenclature \\
\hline$a_{\infty}$ & $=$ speed of sound \\
\hline$b$ & $=$ semichord length \\
\hline$c_{h}, c_{\alpha}$ & $\begin{array}{l}=\text { linear viscous damping coefficients } \\
\text { in plunging and pitching, respectively }\end{array}$ \\
\hline$h, \xi$ & $\begin{array}{l}=\text { plunging displacement and its dimensionless } \\
\text { counterpart }(\equiv h / b), \text { respectively }\end{array}$ \\
\hline$I_{\alpha}$ & $=$ mass moment of inertia per unit span \\
\hline$K_{h}, K_{\alpha}$ & $\begin{array}{l}=\text { linear stiffness coefficients in plunging } \\
\text { and pitching, respectively }\end{array}$ \\
\hline$\hat{K}_{\alpha}, B$ & $\begin{aligned} &= \text { nonlinear stiffness coefficient in pitch and its } \\
& \text { normalized counterpart [Eq. (5)], respectively }\end{aligned}$ \\
\hline$L_{a}, l_{a}, M_{a}, m_{a}$ & $\begin{array}{l}=\text { unsteady lift and moment per unit wing span, } \\
\text { and their dimensionless counterparts, } \\
\left(L_{a} b / m U_{\infty}^{2}\right) \text { and }\left(M_{a} b^{2} / I_{\alpha} U_{\infty}^{2}\right) \text {, respectively }\end{array}$ \\
\hline$M_{C} ; f_{1}, f_{2}$ & $\begin{array}{l}=\text { nonlinear moment control; linear } \\
\text { and nonlinear control gains }\end{array}$ \\
\hline$M_{\alpha}$ & nonlinear restoring moment \\
\hline$M_{\infty}, \lambda$ & $\begin{array}{l}=\text { undisturbed flight Mach number, } U_{\infty} / a_{\infty} \text {, } \\
\text { and its normalized counterpart, } M_{\infty} /\left(\mu \chi_{\alpha} r_{\alpha}\right) \text {, } \\
\text { respectively }\end{array}$ \\
\hline$m$ & $=$ airfoil mass per unit span \\
\hline$p_{\infty}, \rho_{\infty}, a_{\infty}$ & $\begin{array}{l}=\text { pressure, air density, and speed of sound } \\
\text { of the undisturbed flow, respectively }\end{array}$ \\
\hline$q$ & $=$ dynamic pressure, $\frac{1}{2} \rho_{\infty} U_{\infty}^{2}$ \\
\hline$r_{\alpha}$ & $\begin{array}{l}=\text { dimensionless radius of gyration with respect } \\
\text { to the elastic axis, } \sqrt{ }\left(I_{\alpha} / m b^{2}\right)\end{array}$ \\
\hline$S_{\alpha}, \chi_{\alpha}$ & $\begin{array}{l}=\text { static unbalance about the elastic axis } \\
\text { and its dimensionless counterpart, } \\
S_{\alpha} / m b, \text { respectively }\end{array}$ \\
\hline$t, \tau$ & $\begin{array}{l}=\text { time variable and its dimensionless } \\
\text { counterpart, } U_{\infty} t / b, \text { respectively }\end{array}$ \\
\hline$U_{\infty}, V$ & $\begin{aligned}= & \text { freestream speed and its dimensionless } \\
& \text { counterpart, } U_{\infty} / b \omega_{\alpha}, \text { respectively }\end{aligned}$ \\
\hline
\end{tabular}

Received 2 March 2001; revision received 1 March 2002; accepted for publication 7 March 2002. Copyright (C) 2002 by the American Institute of Aeronautics and Astronautics, Inc. All rights reserved. Copies of this paper may be made for personal or internal use, on condition that the copier pay the $\$ 10.00$ per-copy fee to the Copyright Clearance Center, Inc., 222 Rosewood Drive, Danvers, MA 01923; include the code 0731-5090/02 \$10.00 in correspondence with the CCC.

*Visiting Assistant Professor, Department of Engineering Science and Mechanics. Member AIAA.

${ }^{\dagger}$ Professor of Aeronautical and Mechanical Engineering, Department of Engineering Science and Mechanics.

F Senior Research Scientist, Senior Aerospace Engineer, Aeroelasticity Branch, Structures and Materials Competency. Senior Member AIAA.

\begin{tabular}{|c|c|c|}
\hline$V_{F}, \chi_{F}$ & $=$ & speed and frequency of flutter \\
\hline$v_{z}$ & $=$ & $\begin{array}{l}\text { downwash velocity normal } \\
\text { to the lifting surface }\end{array}$ \\
\hline$w$ & $=$ & transverse displacement \\
\hline$x_{\mathrm{EA}}, x_{0}$ & $=$ & $\begin{array}{l}\text { elastic axis position measured from the } \\
\text { leading edge (positive aft) and its } \\
\text { dimensionless counterpart, } x_{\mathrm{EA}} / b, \\
\text { respectively }\end{array}$ \\
\hline$\alpha$ & $=$ & twist angle about the pitch axis \\
\hline$\gamma$ & $=$ & aerodynamic correction factor \\
\hline$\delta_{S}, \delta_{A}, \delta_{C}$ & $=$ & $\begin{array}{l}\text { tracing quantities identifying the structural, } \\
\text { aerodynamic, and nonlinear control terms, } \\
\text { respectively }\end{array}$ \\
\hline$\zeta_{h}, \zeta_{\alpha}$ & $=$ & $\begin{array}{l}\text { damping ratios in plunging and pitching, } \\
c_{h} / 2 m \omega_{h} \text { and } c_{\alpha} / 2 I_{\alpha} \omega_{\alpha} \text {, respectively }\end{array}$ \\
\hline$\kappa$ & $=$ & isentropic gas coefficient \\
\hline$\mu$ & $=$ & dimensionless mass ratio, $m / 4 \rho b^{2}$ \\
\hline$\psi_{1}, \psi_{2}$ & $=$ & $\begin{array}{l}\text { linear and nonlinear normalized control gains, } \\
f_{1} / K_{\alpha} \text { and } f_{2} / K_{\alpha} \text {, respectively }\end{array}$ \\
\hline$\omega_{h}, \omega_{a}, \bar{\omega}$ & $=$ & $\begin{array}{l}\text { uncoupled frequencies of the linearized } \\
\text { aeroelastic system counterpart in plunging } \\
\sqrt{ }\left(K_{h} / m\right) \text {, pitching } \sqrt{ }\left(K_{\alpha} / I_{\alpha}\right) \text {, and frequency } \\
\text { ratio }\left(\omega_{h} / \omega_{\alpha}\right) \text {, respectively }\end{array}$ \\
\hline
\end{tabular}

Superscripts , ,

$=$ time derivative and its dimensionless counterpart, respectively

\section{Introduction}

$\mathbf{H}$ IGH-SPEED and high-performance combat aircraft perform aggressive maneuvers that can result in significant reductions in the flutter speed. Moreover, the tendency to increase structural flexibility and maximum operating speed increases the likelihood of flutter within the aircraft operational envelope. This can jeopardize aircraft performance and dramatically affect its survivability. To prevent such events from occurring, two principal issues need to be addressed: 1) increase of the flutter speed without weight penalties and 2) investigation of the possibilities of converting the unstable limit cycle into a stable limit cycle. The successful accomplishment of the second issue will permit the crossing of the flutter boundary without danger of a catastrophic failure. In such a case, however, structural fatigue becomes a concern.

Before addressing these issues, the search for the aeroelastic instability of lifting surfaces encompasses two basic problems. One of these, based on the linearized aeroelastic equations, allows determination of the flutter boundary. The second one, based on the nonlinear approach to the aeroelastic problem, allows determination 
of the character of the flutter boundary. In this sense, the flutter boundary can feature either benign or catastrophic behavior.

Because of the necessity of avoiding flutter and/or flutter-related airplane performance restrictions, it appears that determination of both the flutter boundary and of its character, that is, catastrophic or benign, and the possibility of controlling both of these present considerable practical importance. The goal of the control is to expand the flight envelope without weight penalties by increasing the flutter speed and to convert the catastrophic flutter into benign flutter.

The concept of catastrophic and benign types of flutter can be found in the specialized literature under different connotations that depend on the particular approach of the problem. The terminology of benign or catastrophic flutter ${ }^{1-4}$ is synonymous with that of stable and unstable limit-cycle oscillation (LCO), ${ }^{5-11}$ also referred to in the literature as supercritical and subcritical Hopf bifurcation ${ }^{12}$ (also Refs. 7, 13, and 14), respectively. The various terminologies related to the character of the flutter boundary and a few sources where these can be found are shown in Table 1. These terminologies are used throughout the paper.

In this study, the issues related to both the increase of the flutter speed and the character of the flutter boundary, as well as of their control, will be addressed. In the aeroelastic governing equations, the various nonlinear effects on which basis is possible to analyze the character of the flutter boundary will be incorporated. An active control methodology capable of expanding the flutter boundary and of converting the unstable LCO into a stable LCO will be implemented. The nonlinearities to be included in the aeroelastic model can be structural, that is, arising from the kinematical equations, ${ }^{7-9,11}$ physical, that is, those involving the constitutive equations, ${ }^{2-4,15,16}$ or aerodynamic appearing in the unsteady aerodynamic equations. ${ }^{2-4,6,17}$ Their contribution can be beneficial (benign flutter boundary) or detrimental (catastrophic flutter boundary). A discussion of this issue in the context of the panel flutter may be found in Refs. 2-5.

The nature of the LCO, which provides important information on the behavior of the aeroelastic system in the vicinity of the flutter boundary, can be examined by the nature of the Hopf bifurcation ${ }^{12}$ of the associated nonlinear aeroelastic system. ${ }^{7,13,14}$ Figure 1 presents

Table 1 Terminologies of the dynamics of nonlinear aeroelastic system

\begin{tabular}{|c|c|}
\hline Terminologies & Selected references \\
\hline $\begin{array}{l}\text { Benign/catastrophic } \\
\text { flutter boundary }\end{array}$ & Bolotin $^{1}$ and Librescu ${ }^{2-4}$ \\
\hline Stable/unstable LCO & $\begin{array}{l}\text { Dowell, }{ }^{5} \text { Friedman and Hanin, }{ }^{6} \\
\text { Lee et al. } .{ }^{7} \text { Lee and Kim, }{ }^{8} \text { Mei, } \\
\text { Morino, }{ }^{10} \text { Strganac et al., }{ }^{11}\end{array}$ \\
\hline $\begin{array}{l}\text { Supercritical/subcritical } \\
\text { H-B }\end{array}$ & $\begin{array}{l}\text { Holmes, }{ }^{13} \text { Lee et al., } \\
\text { Mastroddi and Morino }\end{array}$ \\
\hline
\end{tabular}

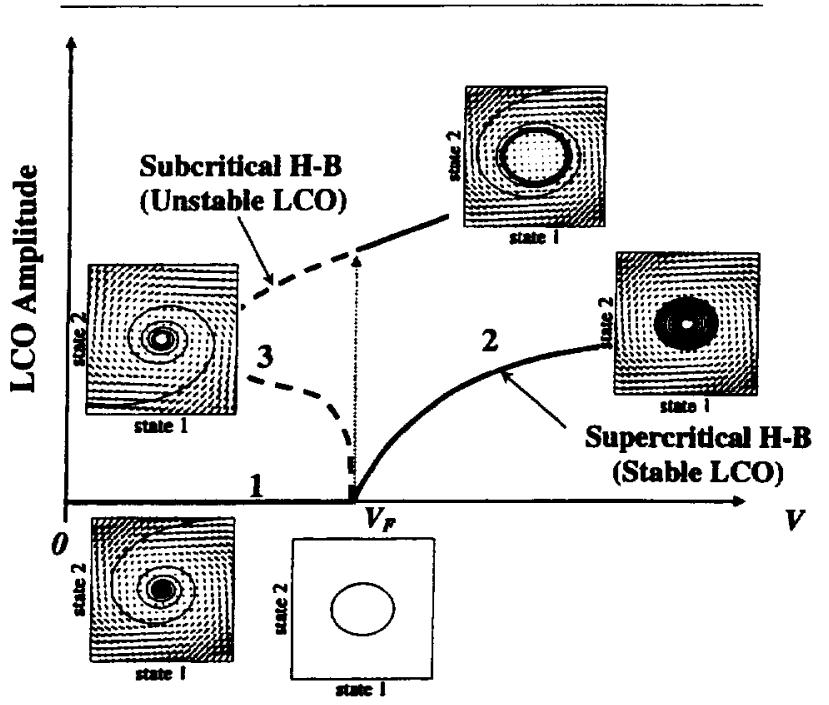

Fig. 1 Character of the flutter boundary in the terms of LCOs amplitudes. several pertinent scenarios; $V=V_{F}$ defines the flutter boundary that can be determined via a linearized analysis. The nonlinear approach to the problem enables one to determine the aeroelastic behavior in the vicinity of the flutter boundary. As a result of the nonlinear analysis, one can determine the aeroelastic behavior for a flight speed lower than the flutter speed $V_{F}$ (curve 1), that is, for $V<V_{F}$, where a subcritical aeroelastic response is experienced. For $V>V_{F}$, the system can exhibit either a stable LCO (supercritical Hopf bifurcation ${ }^{12}$ (H-B), curve 2), or an unstable LCO (subcritical H-B, curve 3).

In this paper, a general approach to the problem of the stability of the LCO of supersonic/hypersonic two-dimensional lifting surfaces is addressed. This methodology enables one to accomplish a parametric study over a large number of parameters that characterize the aeroelastic system. ${ }^{17}$ Literature dealing with the problem of the determination of the flutter boundary of a supersonic/hypersonic wing section and on the nature of the LCO in the presence of both structural and aerodynamic nonlinearities is quite scarce. ${ }^{2-4}$

\section{Nonlinear Model of the Wing Section Incorporating Active Control}

The aeroelastic governing equations of controlled wing section featuring plunging and twisting degrees of freedom, elastically constrained by a linear translational spring and a nonlinear torsional spring exposed to a supersonic/hypersonic flow field are ${ }^{18}$

$$
\begin{gathered}
m \ddot{h}(t)+S_{\alpha} \ddot{\alpha}(t)+c_{h} \dot{h}(t)+K_{h} h(t)=L_{a}(t) \\
S_{\alpha} \ddot{h}(t)+I_{\alpha} \ddot{\alpha}(t)+c_{\alpha} \dot{\alpha}(t)+M_{\alpha}=M_{a}(t)-M_{C}
\end{gathered}
$$

where $h(t)$ is the plunging displacement (positive downward), $\alpha(t)$ is the pitch angle (positive nose up), and the superposed dots denote differentiation with respect to time $t$. Moreover, in Eq. (2)

$$
M_{\alpha}=K_{\alpha} \alpha(t)+\delta_{S} \hat{K}_{\alpha} \alpha^{3}(t)
$$

represents the overall nonlinear restoring moment that involves both the linear and the nonlinear stiffness coefficients, $K_{\alpha}$ and $\hat{K}_{\alpha}$, respectively. The tracer $\delta_{S}$ in Eq. (3) can take the value 1 or 0 depending on whether the nonlinearity is included or ignored, respectively. Within a linear model $\left(\delta_{S}=0\right), M_{\alpha}$ reduces to $K_{\alpha} \alpha(t)$. The nonlinear coefficient $\hat{K}_{\alpha}$ in Eq. (3) can assume positive or negative values. Positive values of $\hat{K}_{\alpha}$ account for hard structural nonlinearities, whereas negative values of $\hat{K}_{\alpha}$ account for soft structural nonlinearities. (Notice that this nonlinearity appears in the present case in the equation relating the restoring moment with the pitch angle and that it has the character of a constitutive equation. For this reason, it would be more appropriate to refer to these as physical nonlinearities.) The active nonlinear control can be represented in terms of the moment $M_{C}$ in a similar functional form as

$$
M_{C}=f_{1} \alpha(t)+\delta_{C} f_{2} \alpha^{3}(t)
$$

In Eq. (4) $f_{1}$ and $f_{2}$ are the linear and nonlinear control gains, respectively. Within a linear active control methodology, the tracer assumes the value $\delta_{C}=0$. Reducing the aeroelastic equations to dimensionless form, we define the parameter $B$ that represents a measure of the degree of the structural nonlinearity of the system and two normalized linear and nonlinear control gain parameters $\psi_{1}$ and $\psi_{2}$, respectively, as

$$
\begin{aligned}
& B=\hat{K}_{\alpha} / K_{\alpha} \\
& \psi_{1}=f_{1} / K_{\alpha} \\
& \psi_{2}=f_{2} / K_{\alpha}
\end{aligned}
$$

Corresponding to $B<0$ or $B>0$, the structural nonlinearities are soft or hard, respectively, whereas for $B=0$, the system is structurally linear.

The nonlinear unsteady aerodynamic lift and moment, from piston theory aerodynamics (PTA), ${ }^{19.20}$ defines pressure on the upper and lower faces of the lifting surface as $p(x, t)=$ $p_{\infty}\left[1+v_{z}(\kappa-1) / 2 a_{\infty}\right]^{2 \kappa /(\kappa-1)}$, where $a_{\infty}^{2}=\kappa p_{\infty} / \rho_{\infty} ; v_{z}$ is the 
downwash velocity normal to the lifting surface expressed as ${ }^{19} v_{z}=$ $-\left(\partial w / \partial t+U_{\infty} \partial w / \partial x\right) \operatorname{sgn} z ; w$ is the transversal displacement of the two-dimensional lifting surface, $w(t)=h(t)+\alpha(t)\left(x-x_{\mathrm{EA}}\right)$; and $\operatorname{sgn} z$ is the sign distribution that assumes the values 1 or -1 for $z>0$ and $z<0$, respectively. In addition, $x_{\mathrm{EA}}=b x_{0}$ is the streamwise position of the pitch axis measured from the leading edge (positive aft).

Retaining in the binomial expansions of $p(x, t)$, the terms up to and including $\left(v_{z} / a_{\infty}\right)^{3}$, yields the pressure formula for the PTA in the third-order approximation $2,3,21$

$$
\begin{aligned}
p / p_{\infty} & =1+\kappa \gamma v_{z} / a_{\infty}+\kappa(\kappa+1) / 4\left(\gamma v_{z} / a_{\infty}\right)^{2} \\
& +\kappa(\kappa+1) / 12\left(\gamma v_{z} / a_{\infty}\right)^{3}
\end{aligned}
$$

The aerodynamic correction factor $\gamma=M_{\infty} / \sqrt{ }\left(M_{\infty}^{2}-1\right)$ enables one to extend the applicability of the PTA to the low supersonic flight-speed range. ${ }^{21,22}$ Equation (6) is valid as long as the transformations through compression and expansion are considered to be isentropic, that is, as long as the shock losses would be insignificant (low-intensity waves). On the other hand, a more general pressure expression, obtained from the theory of oblique shock waves (SWT), that is valid over the entire supersonic/hypersonic range was obtained in Refs. 21 and 22, and it was used in aeroelastic analyses in Refs. 2-4. It is given by

$$
\begin{gathered}
p / p_{\infty}=1+\kappa \gamma v_{z} / a_{\infty}+\kappa(\kappa+1) / 4\left(\gamma v_{z} / a_{\infty}\right)^{2} \\
+\kappa(\kappa+1)^{2} / 32\left(\gamma v_{z} / a_{\infty}\right)^{3}
\end{gathered}
$$

With the exception of the cubic terms, Eqs. (6) and (7) resemble each other. This is explained by the entropy variation appearing in the pressure expansion, beginning with the third-order terms. In contrast to Eq. (6), Eq. (7) encompasses additional features in the sense of 1) taking into account shock losses that occur in the case of strong waves, 2 ) being applicable over a wider range of angles of attack ( $\alpha \leq 30 \mathrm{deg}$ ) and Mach numbers ( $M \geq 1.3$ ) (Refs. 21 and 22), and 3) being applicable to Newtonian speeds $(M \rightarrow \infty, \gamma \rightarrow 1)$. Comparison of results showing the unstable and stable LCOs using the PTA and SWT will be presented next.

The two coefficients of the cubic terms in the two equations differ by $10 \%$ for $k=1.4$, and so, for a more accurate prediction of the character of the flutter instability boundary, it should be included (Ref. 20). However, within a linear stability analysis, the flutter speed evaluated via these two expressions by SWT and PTA does not exhibit any differences.

A comparison of the flutter speed vs flight Mach number obtained from the PTA and SWT, including and discarding the correction factor $\gamma$, is shown in Fig. 2. In addition, in the same figure, the flutter boundary obtained via the use of the linearized supersonic unsteady aerodynamics as provided by Garrick and Rubinow, ${ }^{23}$ is also supplied. In the low-supersonic flight-speed regime, the PTA and $S W T$ with the corrective term provide a rather good agreement

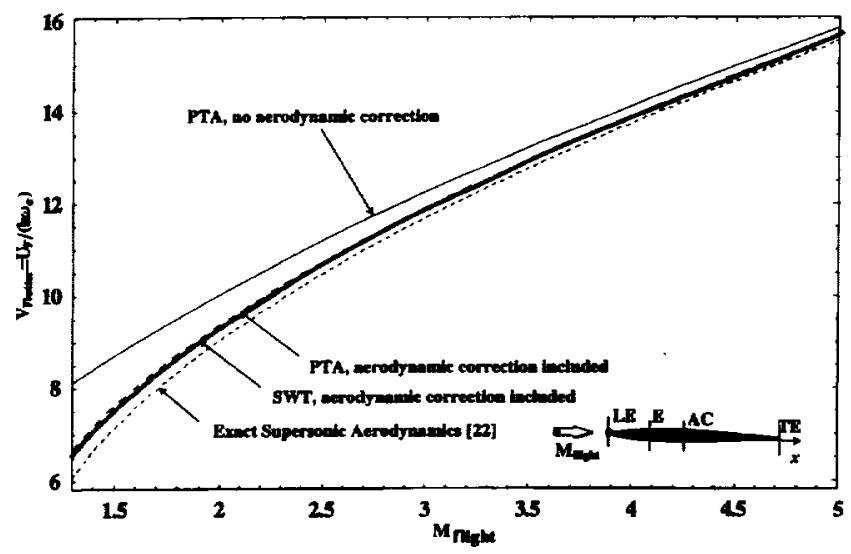

Fig. 2 Comparison of the predictions of the flutter speed vs the flight Mach number when using PTA, SWT, and the exact unsteady supersonic aerodynamies: $\mu=100, \chi_{\alpha}=0.25, \bar{\omega}=1.2, r_{\alpha}=0.5, \zeta_{\alpha}=\zeta_{k}=0$, and $x_{0}=0.5$. with the flutter predictions reached via the supersonic flow theory, ${ }^{23}$ and as a result, this correction should be included. At the same time, for higher supersonic Mach numbers, the differences in the flutter predictions based on the indicated aerodynamic theories practically disappear. In the next developments, unless otherwise stated, PTA will be applied. A comparison of the predictions of the benign and catastrophic character of the flutter boundary, based on these two aerodynamic theories, will be shown subsequently.

When the case of the flow on both surfaces of the airfoil with the speed $U_{\infty}^{+}=U_{\infty}^{-}=U_{\infty}$ is considered, from Eq. (6) the aerodynamic pressure difference $\delta p$ can be expressed as

$$
\begin{aligned}
\left.\delta p\right|_{\mathrm{PTA}} & =\left(4 q / M_{\infty}\right) \gamma\left[\left(w, s / U_{\infty}+w, x\right)\right. \\
+(1+\kappa) / 12 \gamma^{2} M_{\infty}^{2}(w, s & \left.\left(U_{\infty}+w, x\right)^{3}\right]
\end{aligned}
$$

In the next developments, the nonlinear aerodynamic damping in Eq. (8), that is, the terms associated with $(w, t)^{2}$ and $(w, t)^{3}$, will be discarded, and consequently, the cubic nonlinear aerodynamic term reduces to the $\left(w,_{x}\right)^{3}$ only. The study of the implications of the nonlinear aerodynamic damping on the nature of the LCO constitutes an important problem, which is not addressed in this paper.

Next, the nonlinear unsteady aerodynamic lift $L_{a}(t)$ and moment $M_{a}(t)$ per unit wing span can be obtained from the integration of the pressure difference on the upper and lower surfaces of the airfoil:

$$
\begin{gathered}
L_{a}(t)=\int_{0}^{2 b} \delta p \mathrm{~d} x \\
M_{a}(t)=-\int_{0}^{2 b} \delta p\left(x-x_{\mathrm{EA}}\right) \mathrm{d} x
\end{gathered}
$$

The final expressions can be cast in compact form as

$$
\begin{aligned}
& L_{a}(t)=-\left(b U_{\infty} \rho_{\infty} / 3 M_{\infty}\right) \gamma\left\{12 U_{\infty} \alpha(t)\right. \\
& \left.\quad+\delta_{A} M_{\infty}^{2} U_{\infty}(1+\kappa) \gamma^{2} \alpha^{3}(t)+12\left[\dot{h}(t)+\left(b-x_{\mathrm{EA}}\right) \dot{\alpha}(t)\right]\right\} \\
& M_{a}(t)=\left(b U_{\infty} \rho_{\infty} / 3 M_{\infty}\right) \gamma\left\{12 U_{\infty}\left(b-x_{\mathrm{EA}}\right) \alpha(t)\right. \\
& \quad+\delta_{A} M_{\infty}^{2} U_{\infty}\left(b-x_{\mathrm{EA}}\right)(1+\kappa) \gamma^{2} \alpha^{3}(t)+4\left[3\left(b-x_{\mathrm{EA}}\right) \dot{h}(t)\right. \\
& \left.\left.+\left(4 b^{2}-6 b x_{\mathrm{EA}}+3 x_{\mathrm{EA}}^{2}\right) \dot{\alpha}(t)\right]\right\}
\end{aligned}
$$

where $\delta_{A}$ is a tracer that is set equal to $I$ if the aerodynamic nonlinearity is included or set equal to 0 if the aerodynamic nonlinearity is ignored.

As a result, the governing equations (1) and (2) considered in conjunction with Eqs. (10a) and (10b) feature inertial and aerodynamic coupling. Using the dimensionless time $\tau=U_{\infty} t / b$, the system of governing equations can be expressed as

$$
\begin{aligned}
& \xi^{\prime \prime}(\tau)+\chi_{\alpha} \alpha^{\prime \prime}(\tau)+2 \zeta_{h}(\bar{\omega} / V) \xi^{\prime}(\tau)+(\bar{\omega} / V)^{2} \xi(\tau)=l_{a}(\tau) \\
& \left(\chi_{\alpha} / r_{\alpha}^{2}\right) \xi^{\prime \prime}(\tau)+\alpha^{\prime \prime}(\tau)+\left(2 \zeta_{\alpha} / V\right) \alpha^{\prime}(\tau)+1 / V^{2} \alpha(\tau) \\
& \quad+1 / V^{2} B \alpha^{3}(\tau)=m_{a}(\tau)-\psi_{1} / V^{2} \alpha(\tau)-\psi_{2} / V^{2} \alpha^{3}(\tau)
\end{aligned}
$$

In these equations, the dimensionless aerodynamic lift and moment are represented as

$$
\begin{aligned}
l_{a}(\tau) & =-\left(\gamma / 12 \mu M_{\infty}\right)\left\{12 \alpha(\tau)+\delta_{A} M_{\infty}^{2}(1+\kappa) \gamma^{2} \alpha^{3}(\tau)\right. \\
& \left.+12\left[\xi^{\prime}(\tau)+\left(b-x_{\mathrm{EA}}\right) / b \alpha^{\prime}(\tau)\right]\right\} \\
m_{a}(\tau) & =\left(\gamma / 12 \mu M_{\infty}\right)\left(1 / r_{\alpha}^{2}\right)\left\{12\left(b-x_{\mathrm{EA}}\right) / b \alpha(\tau)\right. \\
& +\delta_{A} M_{\infty}^{2}\left(b-x_{\mathrm{EA}}\right) / b(1+\kappa) \gamma^{2} \alpha^{3}(\tau)+4\left[3\left(b-x_{\mathrm{EA}}\right) / b \xi^{\prime}(\tau)\right. \\
& \left.\left.+\left(4 b^{2}-6 b x_{\mathrm{EA}}+3 x_{\mathrm{EA}}^{2}\right) / b^{2} \alpha^{\prime}(\tau)\right]\right\}
\end{aligned}
$$

where $\xi=h / b$ is the dimensionless plunging displacement and the primes denote differentiation with respect to dimensionless time $\tau$. 
When the procedure developed by Bautin ${ }^{24}$ and Lyapunov ${ }^{25}$ is used, pertinent conditions defining the character of the flutter boundary (benign or catastrophic), can be determined. These conditions are expressed in terms of the sign of the Lyapunov first quantity ${ }^{4}$ $L\left(V_{F}\right)$ determined on the flutter boundary. ${ }^{2-4,24}$ Specifically, the inequalities $L\left(V_{F}\right)<0$ and $L\left(V_{F}\right)>0$ define the benign (supercritical) and catastrophic (subcritical) nature of the flutter boundary. The application of Bautin's procedure ${ }^{24}$ requires that the characteristic equations obtained on the flutter boundary exhibit either one root or two roots that are purely imaginary. These conditions are equivalent to the H-B theorem. ${ }^{12}$

The Lyapunov first quantity ${ }^{4} L\left(V_{F}\right)$ corresponding to the nonlinear flutter of the wing section in a supersonic/hypersonic flowfield is derived next and is used to determine the conditions that characterize the nature of the flutter boundary.

The governing equations (11) and (12) are converted to a system of four differential equations in the form ${ }^{2-4,24}$

$$
\frac{\mathrm{d} x_{j}}{\mathrm{~d} \tau}=\sum_{m=1}^{4} a_{m}^{(j)} x_{m}+P_{j}\left(x_{1}, x_{2}, x_{3}, x_{4}\right), \quad j=\overline{1,4}
$$

The functions $P_{j}\left(x_{1}, x_{2}, x_{3}, x_{4}\right)$ include the structural, aerodynamic, and nonlinear control terms that can be cast as

$$
\begin{gathered}
P_{j}\left(x_{1}, x_{2}, x_{3}, x_{4}\right)=\sum_{i=1}^{4} a_{i i}^{(j)} x_{i}^{2}+2 \sum_{\substack{i, l=1 \\
(i \neq l)}}^{4} a_{l i}^{(j)} x_{i} x_{l}+\sum_{i=1}^{4} a_{i i i}^{(j)} x_{i}^{3} \\
+3 \sum_{\substack{i, l=1 \\
(i \neq l)}}^{4} a_{i i l}^{(j)} x_{i}^{2} x_{l}+6 \sum_{\substack{i, j, k=1 \\
(i \neq l \neq k)}}^{4} a_{i l k}^{(j)} x_{i} x_{l} x_{k}
\end{gathered}
$$

For the present case, Eqs. (14) and (15) reduce to a state-space form:

$$
\begin{aligned}
\frac{\mathrm{d} x_{1}}{\mathrm{~d} \tau} & =x_{3} \\
\frac{\mathrm{d} x_{2}}{\mathrm{~d} \tau} & =x_{4} \\
\frac{\mathrm{d} x_{3}}{\mathrm{~d} \tau} & =a_{1}^{(3)} x_{1}(\tau)+a_{2}^{(3)} x_{2}(\tau)+\delta_{A} a_{222}^{(3)} x_{2}^{3}(\tau)+\delta_{S} a_{222}^{(3)} x_{2}^{3}(\tau) \\
& +\delta_{C} a_{222}^{(3)} x_{2}^{3}(\tau)+a_{3}^{(3)} x_{3}(\tau)+a_{4}^{(3)} x_{4}(\tau) \\
\frac{\mathrm{d} x_{4}}{\mathrm{~d} \tau} & =a_{1}^{(4)} x_{1}(\tau)+a_{2}^{(4)} x_{2}(\tau)+\delta_{A} a_{222}^{(4)} x_{2}^{3}(\tau)+\delta_{S} a_{222}^{(4)} x_{2}^{3}(\tau) \\
& +\delta_{C} a_{222}^{(4)} x_{2}^{3}(\tau)+a_{3}^{(4)} x_{3}(\tau)+a_{4}^{(4)} x_{4}(\tau)
\end{aligned}
$$

where

$\xi=x_{1}$

$$
\alpha=x_{2}, \quad \xi^{\prime}=x_{3},
$$$$
\alpha^{\prime}=x_{4}
$$

The linear active control appears in the coefficients $a_{2}^{(3)}$ and $a_{2}^{(4)}$, whereas nonlinear control terms are included in the terms accompanying the tracer $\delta_{C}$. The coefficients of Eqs. (16) are given in Appendix A. When the streamwise position of the pitch axis coincides with that of the midchord, $x_{0}=1$, the nonlinear aerodynamic terms become negligible as the aerodynamic pitching moment vanishes.

When the solution of Eqs. (16) is considered in the form $x_{j}=A_{j} \exp (\omega t)$, the characteristic equation corresponding to the linearized system obtained on the flutter boundary is

$$
\omega^{4}+p \omega^{3}+q \omega^{2}+r \omega+s=0
$$

where

$$
\begin{aligned}
p= & \left\{V \gamma\left[4+3 x_{0}^{2}+6 x_{0}\left(\chi_{\alpha}-1\right)-6 \chi_{\alpha}\right]+3 r_{\alpha}^{2}(V \gamma\right. \\
& \left.\left.+2 M_{\infty} \mu \zeta_{\alpha}+2 M_{\infty} \mu \bar{\omega} \zeta_{h}\right)\right\} / 3 M_{\infty} \mu V\left(r_{\alpha}^{2}-\chi_{\alpha}^{2}\right) \\
q= & \left\{3 M_{\infty} \mu r_{\alpha}^{2}\left\lfloor M_{\infty} \mu\left(1+\bar{\omega}^{2}+\psi_{1}\right)+2 \zeta_{\alpha}\left(V \gamma+2 M_{\infty} \mu \bar{\omega} \zeta_{h}\right)\right]\right. \\
& +V \gamma\left[V\left(\gamma+3 M_{\infty} \mu-3 M_{\infty} \mu \chi_{\alpha}\right)\right. \\
& +8 M_{\infty} \mu \bar{\omega} \zeta_{h}+6 M_{\infty} \mu \bar{\omega} x_{0}^{2} \zeta_{h} \\
& \left.\left.-3 M_{\infty} \mu x_{0}\left(V+4 \bar{\omega} \zeta_{h}\right)\right]\right\} / 3 M_{\infty}^{2} \pi^{2} V^{2}\left(r_{\alpha}^{2}-\chi_{\alpha}^{2}\right)
\end{aligned}
$$

$$
\begin{aligned}
s= & \bar{\omega}^{2}\left[M_{\infty} \mu\left(1+\psi_{1}\right) r_{\alpha}^{2}+V^{2} \gamma\left(x_{0}-1\right)\right] / M_{\infty} \mu V^{4}\left(r_{\alpha}^{2}-\chi_{\alpha}^{2}\right) \\
r= & \left\{3 r_{\alpha}^{2}\left\lfloor 2 M_{\infty} \mu \bar{\omega}^{2} \zeta_{\alpha}+\left(1+\psi_{1}\right)\left(V \gamma+2 M_{\infty} \mu \bar{\omega} \zeta_{h}\right)\right]\right. \\
& +V \gamma \bar{\omega}\left[3 \bar{\omega} x_{0}^{2}-6 x_{0}\left(\bar{\omega}+V \zeta_{h}\right)\right. \\
& \left.\left.+2\left(2 \bar{\omega}+3 V \zeta_{h}\right)\right]\right\} / 3 M_{\infty} \mu V^{3}\left(r_{\alpha}^{2}-\chi_{\alpha}^{2}\right)
\end{aligned}
$$

Three of these parameters, namely, $q, s$, and $r$, include the linear control gain $\psi_{1}$. This has important implications on the possibility of controlling the nature of the LCO and the flutter boundary. For steady-state motion, the equilibrium is stable if the real parts of all of the roots of the characteristic equation are negative. ${ }^{25,26}$ The analysis of the roots of this equation leads to the Routh-Hurwitz ( $\mathrm{R}-\mathrm{H}$ ) conditions which define the parameter bound for the stability of the system. The R-H conditions reduce to the inequalities $p>0$, $q>0, r>0, s>0$, and $\Re=p q r-s p^{2}-r^{2}>0$. For the aeroelastic stability problems, for which the condition $\Delta_{1}=s p / r+p^{2} / 4>0$ should be satisfied, the roots of the characteristic equation on the critical flutter boundary $\Re=0$, are given by

$$
\begin{gathered}
\omega_{1,2}= \pm i c, \quad \omega_{3,4}=-\varepsilon \pm i n \quad \text { where } \quad i=\sqrt{-1} \\
c^{2}=r / p, \quad \varepsilon=+p / 2 \\
n^{2}=s p / r-p^{2} / 4, \quad n>0
\end{gathered}
$$

Equation (19a) is a necessary requirement for the determination of $L\left(V_{F}\right)$ and constitutes a statement of the applicability of the H-B theorem as well. ${ }^{12,24}$

For sufficiently small values of the speed $V$, all of the roots of the characteristic equation are in the left-hand side of the complex plane, and the zero solution of the system is asymptotically stable. For the value $V=V_{F}$, two roots of the characteristic equations are purely imaginary, and the remaining two are complex conjugate and remain in the left-hand side of the complex plane (critical flutter velocity).

To identify the benign and catastrophic character of the flutter boundary, it is necessary to solve the stability problem for the system of equations in state-space form in the critical case of a pair of pure imaginary roots. The expression of the flutter boundary $\mathfrak{R}$ for the wing section is

$$
\begin{aligned}
\Re= & \left\{3 M _ { \infty } \mu \overline { \omega } ^ { 2 } [ V ^ { 2 } \gamma ( x _ { 0 } - 1 ) - M _ { \infty } \mu ( 1 + \psi _ { 1 } ) r _ { \alpha } ^ { 2 } ] \left\{3 r_{\alpha}^{2}(V \gamma\right.\right. \\
& \left.+2 M_{\infty} \mu \zeta_{\alpha}+2 M_{\infty} \mu \bar{\omega} \zeta_{h}\right)+V \gamma\left[4+3 x_{0}^{2}+6 x_{0}\left(\chi_{\alpha}-1\right)\right. \\
& \left.\left.-6 \chi_{\alpha}\right]\right\}^{2}-3 M_{\infty}^{2} \mu^{2}\left\{3 r _ { \alpha } ^ { 2 } \left[2 M_{\infty} \mu \bar{\omega}^{2} \zeta_{\alpha}+\left(1+\psi_{1}\right)(V \gamma\right.\right. \\
& \left.\left.+2 M_{\infty} \mu \bar{\omega} \zeta_{h}\right)\right]+V \gamma \bar{\omega}\left[4 \bar{\omega}+3 \bar{\omega} x_{0}^{2}+6 V \zeta_{h}-6 x_{0}(\bar{\omega}\right. \\
& \left.\left.\left.+V \zeta_{h}\right)\right]\right\}^{2}\left(r_{\alpha}^{2}-\chi_{\alpha}^{2}\right)+\left\{3 r _ { \alpha } ^ { 2 } \left[2 M_{\infty} \mu \bar{\omega}^{2} \zeta_{\alpha}+\left(1+\psi_{1}\right)(V \gamma\right.\right. \\
& \left.\left.+2 M_{\infty} \mu \bar{\omega} \zeta_{h}\right)\right]+V \gamma \bar{\omega}\left[4 \bar{\omega}+3 \bar{\omega} x_{0}^{2}+6 V \zeta_{h}\right. \\
& \left.\left.-6 x_{0}\left(\bar{\omega}+V \zeta_{h}\right)\right]\right\}\left\{3 r_{\alpha}^{2}\left(V \gamma+2 M_{\infty} \mu \zeta_{\alpha}+2 M_{\infty} \mu \bar{\omega} \zeta_{h}\right)\right. \\
& \left.+V \gamma\left[4+3 x_{0}^{2}+6 x_{0}\left(\chi_{\alpha}-1\right)-6 \chi_{\alpha}\right]\right\}\left\{3 M _ { \infty } \mu r _ { \alpha } ^ { 2 } \left[M_{\infty} \mu(1\right.\right. \\
& \left.\left.+\bar{\omega}^{2}+\psi_{1}\right)+2 \zeta_{\alpha}\left(V \gamma+2 M_{\infty} \mu \bar{\omega} \zeta\right)\right]+V \gamma\left[8 M_{\infty} \mu \bar{\omega} \zeta_{h}\right. \\
& +6 M_{\infty} \mu \bar{\omega} x_{0}^{2} \zeta_{h}-3 M_{\infty} \mu x_{0}\left(V+4 \bar{\omega} \zeta_{h}\right)+V\left(\gamma+3 M_{\infty} \mu\right. \\
& \left.\left.\left.\left.-3 M_{\infty} \mu \chi_{\alpha}\right)\right]\right\}\right\} / 27 M^{4} V^{6} \mu^{4}\left(r_{\alpha}^{2}-\chi_{\alpha}^{2}\right)^{3}=0
\end{aligned}
$$

Notice that this expression is general and includes the relationship between the flutter speed and the flutter frequency parameters evaluated on $\Re=0$ in terms of the basic geometrical and flight parameters. In the particular case in which the structural damping ratios 


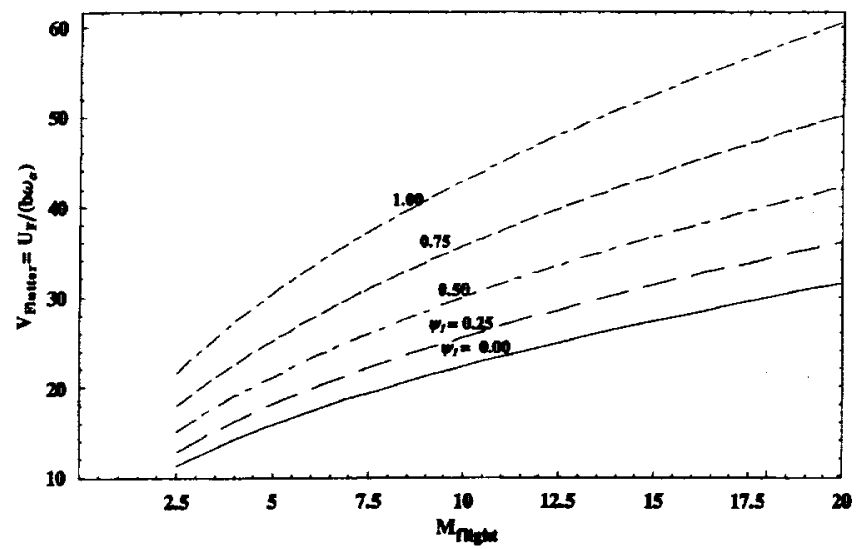

Fig. 3 Flutter speed v8 flight Mach number; influence of the linear control gain $\psi_{1}$.

in plunging and pitching are ignored and $\gamma \Rightarrow 1$, the expressions of flutter frequency $\chi_{F}$ and flutter speed $V_{F}$ reduce to

$$
\begin{aligned}
\chi_{F}= & \left(\omega_{\alpha} / \omega\right)_{F}^{2}=\left[r_{\alpha}^{2}+\left(1-x_{0}\right)^{2}+\frac{1}{3}\right. \\
& \left.-2 \chi_{\alpha}\left(1-x_{0}\right)\right] /\left\{\left(1+\psi_{1}\right) r_{\alpha}^{2}+\vec{\omega}^{2}\left[\left(1-x_{0}\right)^{2}+\frac{1}{3}\right]\right\}
\end{aligned}
$$

and

$V_{F}=\frac{U_{F}}{b \omega_{\alpha}}=\frac{\mu M}{\sqrt{\chi_{F}}}$

$\times \sqrt{\frac{\chi_{\alpha}^{2}-\left(\vec{\omega}^{2} \chi_{F}-1\right) r_{\alpha}^{2}\left(\chi_{F}-1\right)}{\mu M\left[\left(1+\psi_{1}\right) \chi_{\alpha}+\left(1-x_{0}\right)\left(\bar{\omega}^{2} \chi_{F}-\left(1+\psi_{1}\right)\right)\right]-\frac{1}{3}\left(1+\psi_{1}\right)}}$

respectively.

Equations (21) and (22) represent the dimensionless flutter frequency and flutter speed of the actively controlled system. These constitute the extension of the equations obtained by Ashley and Zartarian, ${ }^{19}$ to include active control.

In Fig. 3, the dependence of the dimensionless flutter speed as a function of the Mach number for selected values of the feedback gain $\psi_{1}$ is presented. It clearly appears that, with the increase of the linear control gain, an increase of the flutter speed is experienced. Moreover, as values of $\psi_{1}$ are increased, the efficiency of the active control is increased.

The expression of the Lyapunov first quantity ${ }^{4}$ is given in closed form in Refs. 2 and 4. For the present case, the Lyapunov first quantity ${ }^{4}$ is expressed in terms of the coefficients $A_{k l s}^{(j)}$ as (Appendix B)

$$
L\left(V_{F}\right)=(3 \pi / 4 c)\left(A_{111}^{(1)}+A_{222}^{(2)}+A_{112}^{(2)}+A_{122}^{(1)}\right)
$$

The terms in the parentheses of Eq. (23) are expressed via the coefficients $a_{k l s}^{(j)}$ appearing in Eqs. (15) as

$$
A_{k l s}^{(j)}=\left(1 / \Delta_{0}\right)\left(\hat{\alpha}_{j 3} a_{222}^{(3)} \alpha_{2 k} \alpha_{2 /} \alpha_{2 s}+\hat{\alpha}_{j 4} a_{222}^{(4)} \alpha_{2 k} \alpha_{2 /} \alpha_{2 s}\right)
$$

whereas the coefficients of the system of Eq. (24) are

$$
\begin{aligned}
a_{222}^{(3)} & =\left(\delta_{S} B+\delta_{C} \psi_{2}\right) \frac{r_{\alpha}^{2} \chi_{\alpha}}{V_{F}^{2}\left(r_{\alpha}^{2}-\chi_{\alpha}\right)} \\
& -\delta_{A} \frac{M_{\infty}(1+\kappa) \gamma^{3}\left[\left(x_{0}-1\right) \chi_{\alpha}+r_{\alpha}^{2}\right]}{12 \mu\left(r_{\alpha}^{2}-\chi_{\alpha}\right)} \\
& =\left(\delta_{S} S_{\mathrm{NL}}+\delta_{C} C_{\mathrm{NL}}\right) \chi_{\alpha}-\delta_{A} A_{\mathrm{NL}}\left[\left(x_{0}-1\right) \chi_{\alpha}+r_{\alpha}^{2}\right] \\
a_{222}^{(4)} & =-\left(\delta_{S} B+\delta_{C} \psi_{2}\right) \frac{r_{\alpha}^{2}}{V_{F}^{2}\left(r_{\alpha}^{2}-\chi_{\alpha}\right)} \\
& +\delta_{A} \frac{M_{\infty}(1+\kappa) \gamma^{3}\left(x_{0}-1+\chi_{\alpha}\right)}{12 \mu\left(r_{\alpha}^{2}-\chi_{\alpha}\right)} \\
& =-\left(\delta_{S} S_{\mathrm{NL}}+\delta_{C} C_{\mathrm{NL}}\right)+\delta_{\mathrm{A}} A_{\mathrm{NL}}\left(x_{0}-1+\chi_{\alpha}\right)
\end{aligned}
$$

The dimensionless structural, aerodynamic, and control nonlinearities are defined as

$$
\begin{aligned}
S_{\mathrm{NL}} & =B \frac{r_{\alpha}^{2}}{V_{F}^{2}\left(r_{\alpha}^{2}-\chi_{\alpha}\right)} \\
A_{\mathrm{NL}} & =\frac{M_{\infty}(1+\kappa) \gamma^{3}}{12 \mu\left(r_{\alpha}^{2}-\chi_{\alpha}\right)} \\
C_{\mathrm{NL}} & =\psi_{2} \frac{r_{\alpha}^{2}}{V_{F}^{2}\left(r_{\alpha}^{2}-\chi_{\alpha}\right)}
\end{aligned}
$$

where $V_{F}$ is the flutter speed. Upon defining $\Im_{j p}=\left[\alpha_{j p}\right]^{-1}$ and having in view that, according to Eqs. (19b) $c>0$, the Lyapunov first quantity ${ }^{4}$ reduces to

$$
\begin{aligned}
& L\left(V_{F}\right)=\left(\Im_{13} a_{222}^{(3)}+\Im_{14} a_{222}^{(4)}\right) \alpha_{21}^{3}+\left(\Im_{23} a_{222}^{(3)}+\Im_{24} a_{222}^{(4)}\right) \alpha_{22}^{3} \\
& \quad+\left(\Im_{23} a_{222}^{(3)}+\Im_{24} a_{222}^{(4)}\right) \alpha_{22} \alpha_{12}^{2}+\left(\Im_{13} a_{222}^{(3)}+\Im_{14} a_{222}^{(4)}\right) \alpha_{12} \alpha_{22}^{2}
\end{aligned}
$$

When the expressions of $S_{\mathrm{NL}}, A_{\mathrm{NL}}$, and $C_{\mathrm{NL}}$ [Eqs. (26)] are used and the procedure devised in Refs. $2-4$ is applied, the benign or catastrophic character of the flutter boundary defined as $L\left(V_{F}\right)<0$ or $L\left(V_{F}\right)>0$, respectively. These conditions can be restated as 4

$$
V_{F}<V_{r}
$$

or

$$
V_{F}>V_{r}
$$

where

$$
V_{r}^{2}=A_{1} / A_{2}
$$

In Eq. (28) the parameter $A_{1}$ includes the structural nonlinearities and the nonlinear control gain parameter, whereas $A_{2}$ includes the aerodynamic nonlinearities. Their expressions are provided in Appendix $\mathrm{C}$.

In the absence of the nonlinear control and for $B<0$ (soft structural nonlinearities), $V_{r}$ is negative. The relation $V_{F}>V_{r}$ corresponds to the catastrophic flutter boundary (unstable LCO) and occurs for any supersonic flight Mach number. For this case, an unstable LCO is experienced even in the presence of the linear control. On the other hand, for $B>0$ (hard structural nonlinearities), the transition from benign to catastrophic flutter (from stable to the unstable LCO) occurs at an increased flight Mach number in the presence of the linear control.

As a special case, for $x_{0}=1, \mathrm{Eq}$. (23) reduces to the following form:

$$
\begin{aligned}
& L\left(V_{F}\right)=\left(\delta_{S} S_{\mathrm{NL}}+\delta_{C} C_{\mathrm{NL}}\right)\left\lfloor\left(\Im_{13} \chi_{\alpha}-\Im_{14}\right) \alpha_{21}^{3}+\left(\Im_{23} \chi_{\alpha}-\Im_{24}\right) \alpha_{22}^{3}\right. \\
& \left.+\left(\Im_{23} \chi_{\alpha}-\Im_{24}\right) \alpha_{22} \alpha_{12}^{2}+\left(\Im_{13} \chi_{\alpha}-\Im_{14}\right) \alpha_{12} \alpha_{22}^{2}\right\rfloor \\
& \quad-\delta_{A} A_{\mathrm{NL}}\left[\left(\Im_{13} r_{\alpha}^{2}-\Im_{14} \chi_{\alpha}\right) \alpha_{21}^{3}+\left(\Im_{23} r_{\alpha}^{2}-\Im_{24} \chi_{\alpha}\right) \alpha_{22}^{3}\right. \\
& \left.+\left(\Im_{23} r_{\alpha}^{2}-\Im_{24} \chi_{\alpha}\right) \alpha_{22} \alpha_{12}^{2}+\left(\Im_{13} r_{\alpha}^{2}-\Im_{14} \chi_{\alpha}\right) \alpha_{12} \alpha_{22}^{2}\right]
\end{aligned}
$$

In this case, a decrease of the influence of the aerodynamic nonlinearities on the aeroelastic system is experienced.

\section{Stability in the Presence of Active Control}

To enhance understanding of the effect of the active control on the character of the flutter boundary, some explanations are provided in Figs. $4 \mathrm{a}$ and $4 \mathrm{~b}$. In Fig. 4a, the intersection point between the two curves $V_{F}$ and $V_{r}$ separates the benign flutter boundary (stable LCO) characterized by $V_{F}<V_{r}$ from the catastrophic flutter boundary (unstable LCO) defined by $V_{F}>V_{r}$. For these cases; a change in the sign of the Lyapunov first quantity ${ }^{4} L\left(V_{F}\right)$ occurs (Fig. 4b). In this context, the following four possible scenarios are distinguished: 1) for $V<V_{F}$, as time unfolds, a decay of the motion amplitude 


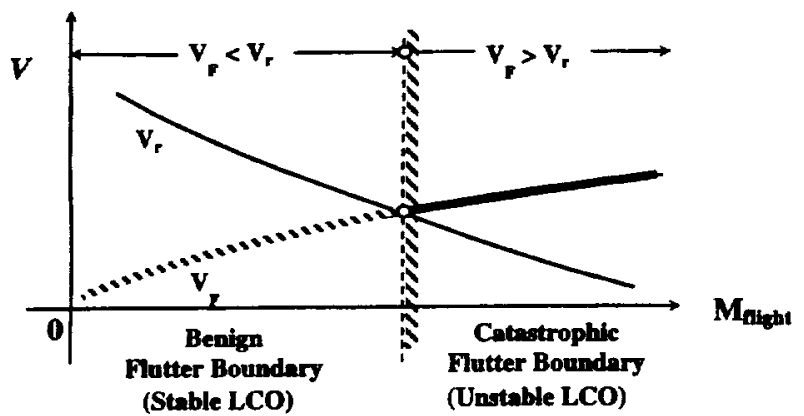

a) In the $\left(V-M_{\text {atpht }}\right)$ plane

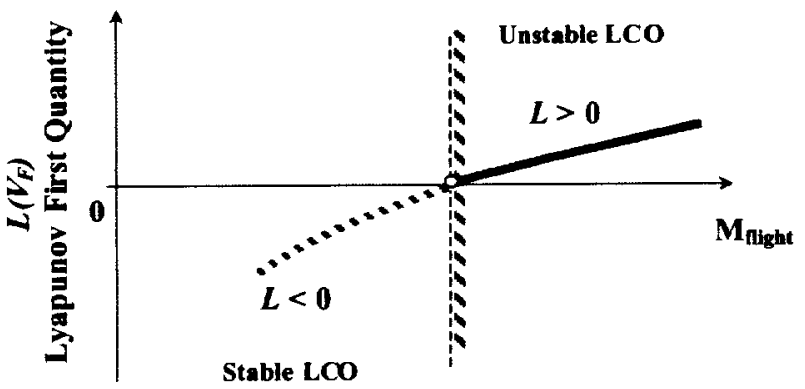

b) In the ( $\left.L-M_{\text {night }}\right)$ plane

Fig. 4 Generic representation of the flutter boundary, $L<0$ corresponds to $V_{r}>V_{F}$ and vice versa.

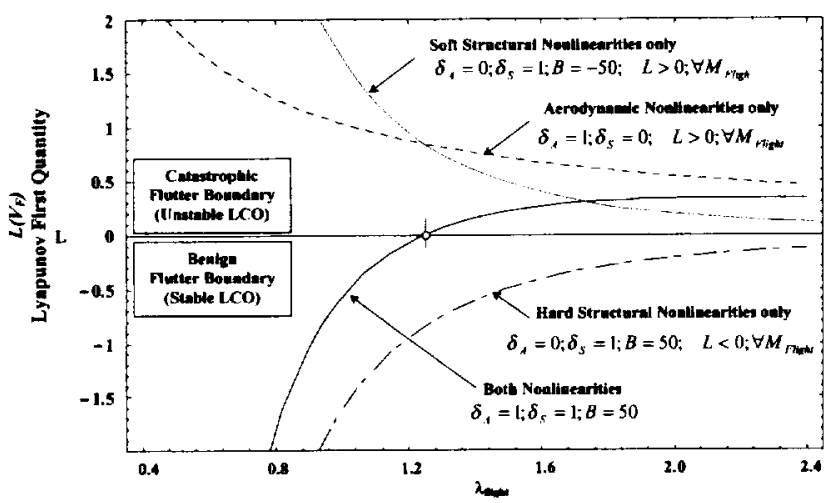

Fig. 5 Influence of the structural and aerodynamical nonlinearities on the Lyapunov first quantity ${ }^{4} L$ for the uncontrolled wing section.

is experienced, indicative of subcritical response; 2) for $V=V_{F}$, the center limit cycle occurs, indicative of a periodic orbit; 3) for $V_{r}>V_{F}$, the LCO is stable; and 4) for $V_{F}>V_{r}$, the LCO is unstable.

The parameters for the simulations, are chosen as $\mu=100$, $\chi_{\alpha}=0.25, \bar{\omega}=1.2, r_{\alpha}=0.5, \zeta_{\alpha}=\zeta_{h}=0, x_{0}=0.5, \gamma=1, \kappa=1.4$, $\delta_{A}=\delta_{S}=\delta_{C}=1$, and $B=50$.

The effect of structural nonlinearities on the character of the flutter boundary is studied in terms of the nonlinear parameter $B$ [Eqs. (3) and (5a)]. For the present simulation, the aeroelastic system appears to be characterized by a catastrophic flutter boundary in the upper half-plane (unstable LCO) and by a benign flutter boundary in the lower half-plane (stable LCO). In Fig. 5 , the Lyapunov first quantity ${ }^{4}$ $L\left(V_{F}\right)$ for cases in which the soft and hard structural and aerodynamic nonlinearities are involved is presented. It appears that in the presence of only the aerodynamic nonlinearities, Lyapunov first quantity becomes positive for any flight Mach number. This result reveals that aerodynamic nonlinearity induces a catastrophic flutter boundary, implying that a subcritical H-B occurs. On the other hand, in the presence of hard structural nonlinearities only, the opposite situation is experienced. At relatively moderate supersonic flight Mach numbers, a benign flutter boundary is encountered, which becomes catastrophic as the Mach number is increased. This implies that for higher Mach numbers the effects of the aerodynamic nonlinearities become prevalent. It is also shown that in the case of high
Mach numbers, the neglect of nonlinear aerodynamic terms yields inadvertent predictions related to the character of the flutter boundary. Moreover, when the aerodynamic nonlinearities are discarded $\left(\delta_{A}=0\right)$ for any flight Mach number, the flutter boundary is benign or catastrophic, depending on whether hard $(B>0)$ or soft $(B<0)$ structural nonlinearities are present, respectively:

The influence of the hard structural nonlinearities, in conjunction with the aerodynamic nonlinearities $\left(\delta_{A}=1\right)$, for the controlled/ uncontrolled system is presented in Fig. 6. The dotted lines identify the cases in which hard structural nonlinearity are included $(B=50)$, whereas the solid lines identify the cases in which the structural nonlinearities are ignored $(B=0)$. The control acts in both situations toward the stabilization of the system. Also, the unstable LCO that occurs when only the aerodynamic nonlinearities are considered can be converted to a stable LCO.

Figures 7 and 8 show that soft structural nonlinearities $(B=-10)$ result in a catastrophic flutter boundary and that via a combined

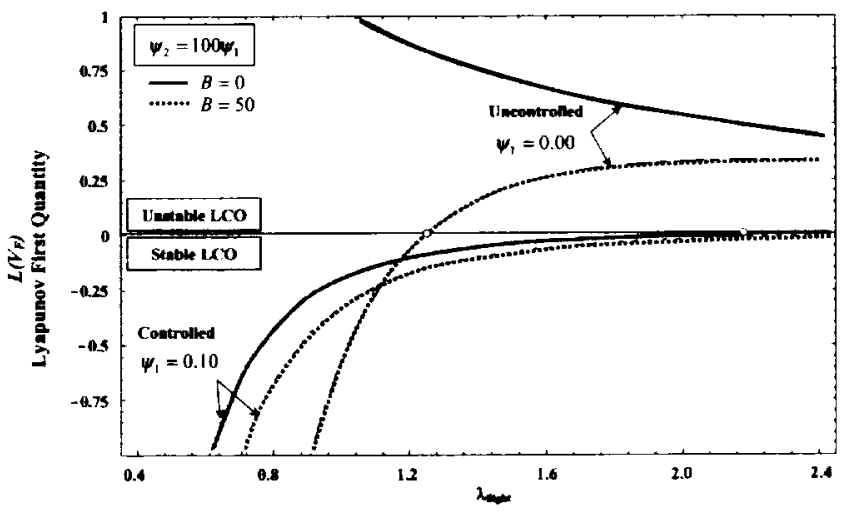

Fig. 6 Infiuence of the structural and aerodynamical nonlinearitles on the Lyapunov first quantity ${ }^{4} L$ in the presence/absence of linear and nonlinear active control gains. Aerodynamic nonlinearities retained.

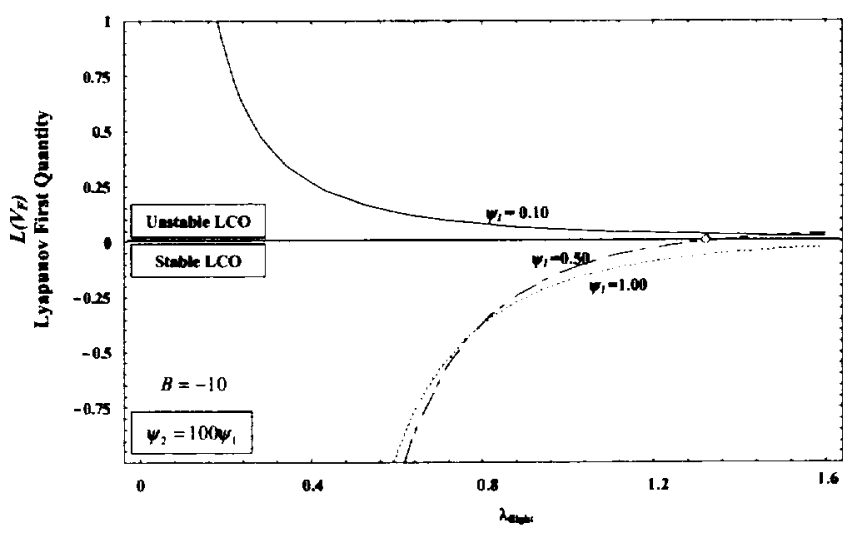

Fig. 7 Conversion of the unstable to stable $\mathrm{LCO}$ via nonlinear active control, for system encompassing structural soft nonlinearity.

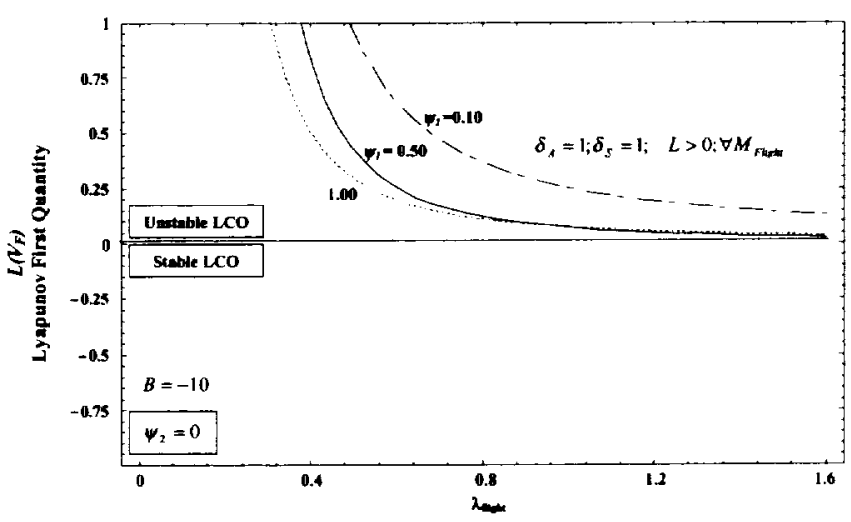

Fig. 8 Unstable LCO for systems encompassing structural soft nonlinearity, aerodynamical nonlinearities, and linear active control. 
active control $\left(\psi_{1}=0.5, \psi_{2}=100 \psi_{1}\right)$ the unstable LCO can become stable (Fig. 7). Moreover, it clearly appears that, when soft structural $(B<0)$ and aerodynamic nonlinearities are present, the linear active control $\left(\forall \psi_{1}>0, \psi_{2}=0\right)$ cannot change the character of the flutter boundary (Fig. 8).

From Eq. (23), which defines the Lyapunov first quantity, ${ }^{4}$ the benign flutter boundary is expressed in closed form. When Eqs. (28a) and (28b) are used, the character of the flutter boundary is examined and has been plotted in Figs. 9-11. Each of Figs. 9-11, display in the plane $\left(V, \lambda_{\text {flight }}\right)$ the benign and catastrophic characters of the flutter boundary for the actively controlled wing section where $\lambda=M_{\infty} /\left(\mu \chi_{\alpha} r_{\alpha}\right)$. The corresponding Lyapunov first quantity is shown in Figs. $12-14$ in the plane $\left(L, \lambda_{\text {fight }}\right)$. In Figs. 9 15 , the aerodynamic and hard structural nonlinearities have been included, $\delta_{A}=1$ and $\delta_{S}=1 ; B=50$. In Figs. 9-15, the transition from catastrophic to benign flutter is shown. Figures 9-15 help one

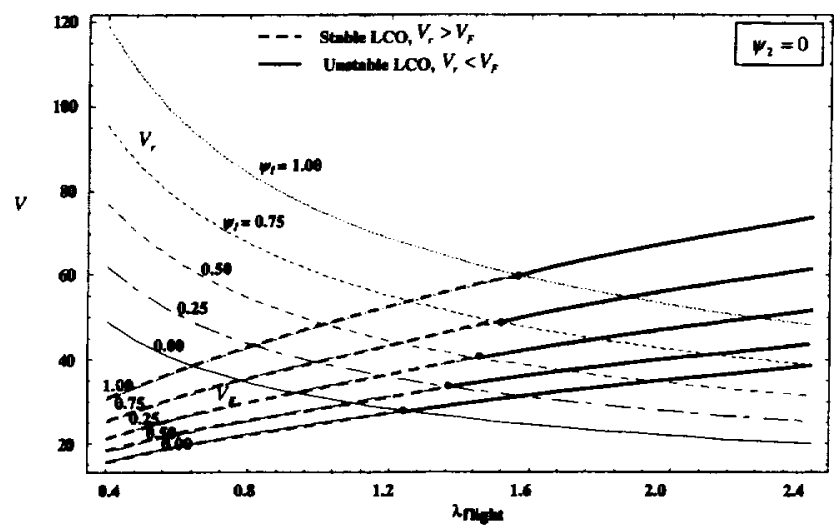

Fig 9 Stable and unstable LCOs in the presence of linear control.

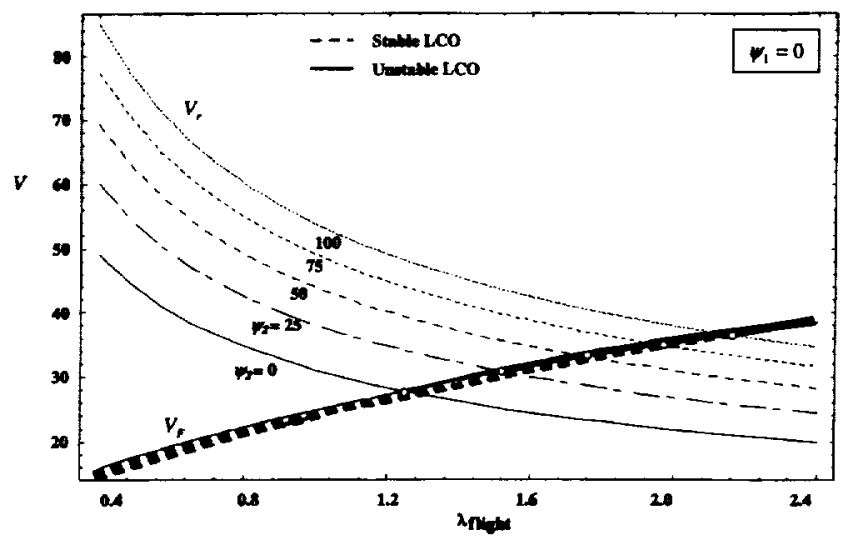

Fig. 10 Stable and unstable LCOs in the presence of nonlinear control.

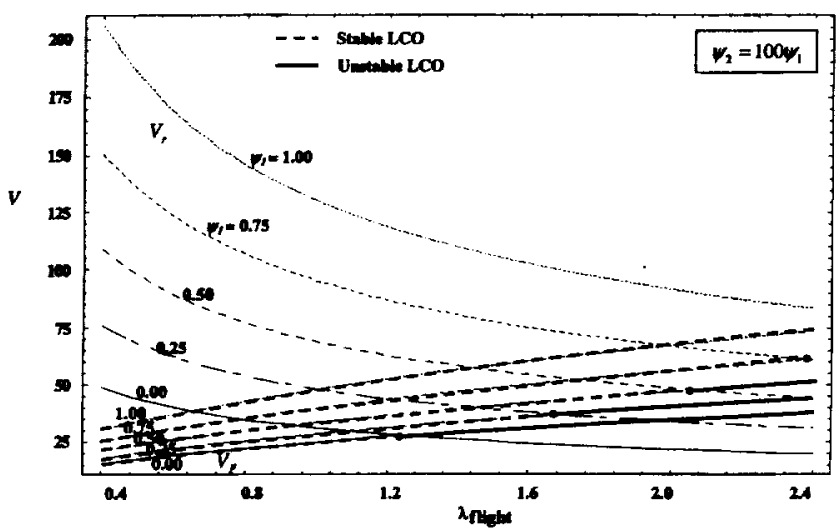

Fig. 11 Stable and unstable LCOs in the presence of linear and nonlinear controls.

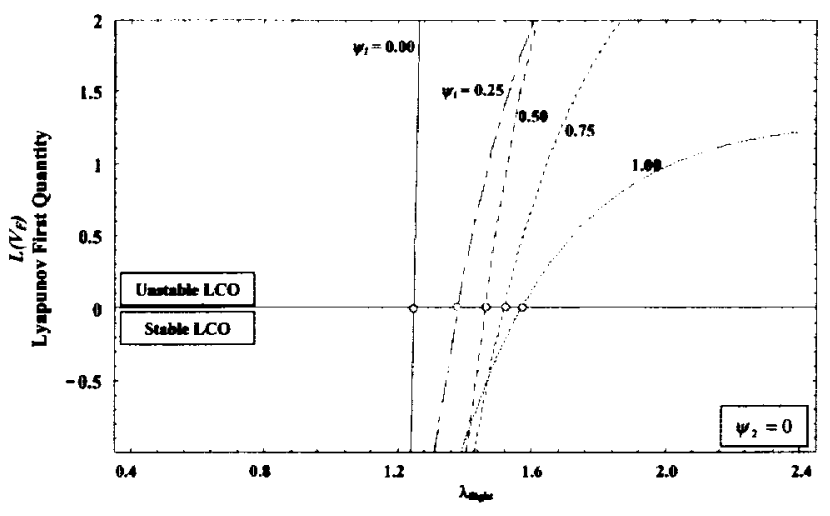

Fig. 12 Influence of the linear control on the Lyapunov first quantity $L$.

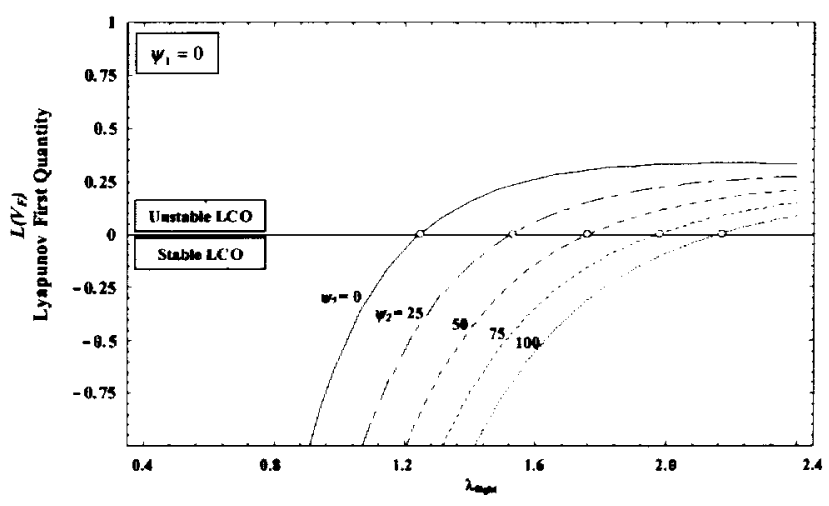

Fig. 13 Influence of the nonlinear control on the Lyapunov first quantity $y^{4} L$.

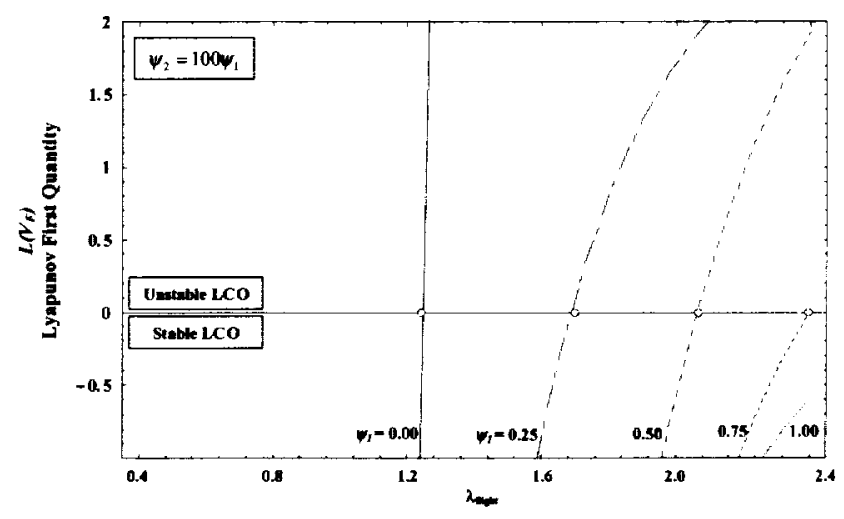

Fig. 14 Influence of the linear and nonlinear controls on the Lyapunov first quantity $L$.

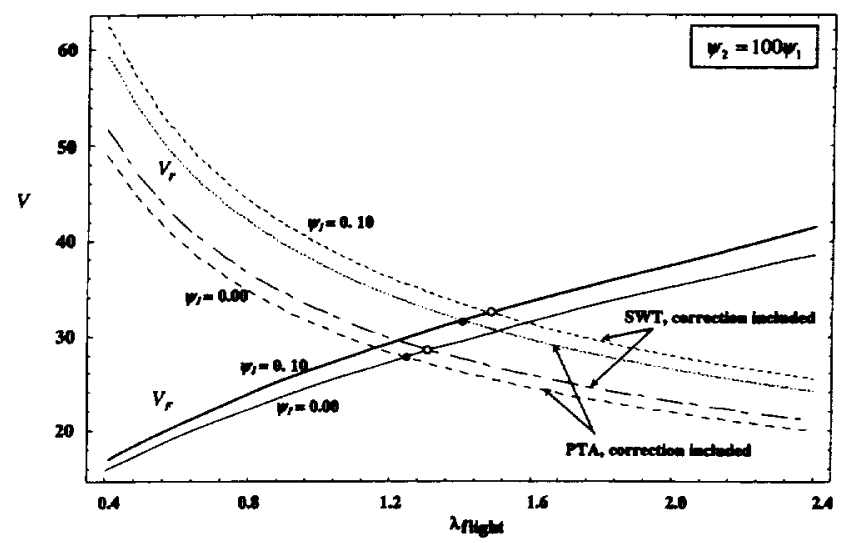

Fig. 15 Stable and unstable LCOs with and without control in presence of both nonlinearities; comparison between SWT and PTA prediction. 
Table 2 Stable and unstable LCOs for PTA and SWT

\begin{tabular}{|c|c|c|c|c|c|c|c|}
\hline \multirow{2}{*}{$\frac{\lambda_{\text {ftight }}}{1.200}$} & \multirow{2}{*}{$\frac{\lambda_{\text {flunter }}}{2.182}$} & \multirow{2}{*}{$\frac{\lambda_{r}, \text { PTA }}{2.263}$} & \multirow{2}{*}{$\frac{\lambda_{r}, \mathrm{SWT}}{2.386}$} & \multicolumn{2}{|c|}{$\begin{array}{c}10^{10} L\left(V_{F}\right) \\
\text { PTA }\end{array}$} & \multicolumn{2}{|c|}{$\begin{array}{c}10^{10} L\left(V_{F}\right) \\
\text { SWT }\end{array}$} \\
\hline & & & & -6.679 & & -15.437 & \\
\hline 1.208 & 2.189 & 2.256 & 2.378 & -5.402 & & -14.107 & \\
\hline 1.216 & 2.196 & 2.248 & 2.370 & -4.164 & $\frac{2}{0}$ & -12.817 & \\
\hline 1.224 & 2.203 & 2.241 & 2.362 & -2.965 & & -11.566 & \\
\hline 1.232 & 2.211 & 2.234 & 2.355 & -1.803 & & -10.352 & \\
\hline 1.240 & 2.218 & 2.226 & 2.347 & -0.676 & $\gamma$ & -9.175 & $\div 0$ \\
\hline 1.248 & 2.225 & 2.219 & 2.339 & 0.416 & & -8.033 & 过 \\
\hline 1.256 & 2.232 & 2.212 & 2.332 & 1.474 & & -6.925 & \\
\hline 1.264 & 2.239 & 2.205 & 2.325 & 2.501 & & -5.849 & \\
\hline 1.272 & 2.246 & 2.198 & 2.317 & 3.496 & & -4.806 & \\
\hline 1.280 & 2.253 & 2.191 & 2.310 & 4.461 & & -3.793 & \\
\hline 1.288 & 2.260 & 2.185 & 2.303 & 5.396 & $\ddot{0}$ & -2.811 & \\
\hline 1.296 & 2.267 & 2.178 & 2.296 & 6.304 & & -1.857 & \\
\hline 1.304 & 2.274 & 2.171 & 2.289 & 7.183 & 5 & -0.930 & \\
\hline 1.312 & 2.281 & 2.164 & 2.282 & 8.037 & & -0.031 & \\
\hline 1.320 & 2.288 & 2.158 & 2.275 & 8.865 & & 0.842 & $x$ \\
\hline 1.328 & 2.295 & 2.151 & 2.268 & 9.668 & & 1.689 & 읨 \\
\hline 1.336 & 2.302 & 2.145 & 2.261 & 10.447 & & 2.512 & \\
\hline 1.344 & 2.309 & 2.139 & 2.254 & 11.202 & & 3.312 & 氙 \\
\hline 1.352 & 2.316 & 2.132 & 2.248 & 11.935 & & 4.088 & \\
\hline 1.360 & 2.322 & 2.126 & 2.241 & 12.646 & & 4.842 & \\
\hline
\end{tabular}

to understand the behavior of the aeroelastic system in the presence of linear and nonlinear active control.

The stable and unstable characters of the LCOs via the use of the two different aerodynamic theories (PTA and SWT) are presented in Fig. 15. From Fig. 15 and Table 2, one can infer that the transition from the stable LCO to the unstable LCO occurs at slightly lower Mach numbers (less then 3\%) for the PTA as compared to those predicted by the SWT. This implies that the PTA provides conservative results as compared to the SWT.

\section{Conclusions}

An original LCO analysis was presented. In contradistinction with the ones generally used in the literature, where the character of the flutter boundary is determined from the path variation amplitude of displacement quantities, in the present approach this information is obtained via the Lyapunov first quantity. ${ }^{4}$

It was shown that in some circumstances, the aerodynamic and hard structural nonlinearities contribute in different ways to the determination of stable or unstable LCOs. It was shown that the hard structural nonlinearities result in a stable LCO, whereas the soft structural nonlinearities result in an unstable LCO. At high flight Mach numbers, the aerodynamic nonlinearities are primary contributors to the destabilization of the aeroelastic system. This implies that with increasing the hypersonic flight speed, when the aerodynamic nonlinearities become prevalent, an unstable LCO occurs irrespective of the presence of hard structural nonlinearities. On the other hand, soft structural nonlinearities $(B<0)$ contribute in the same sense, as the aerodynamic nonlinearities, to the unstable LCO. It is also shown that active control can be used to increase the flutter speed and to convert the catastrophic flutter boundary into a benign flutter boundary and/or to shift the transition between these two states toward higher flight Mach numbers.

The issue of generating the active control moment was not addressed. It is the authors' belief that this can be produced via a device operating similarly to a spring, whose linear and nonlinear characteristics can be controlled, but additional analysis are required to confirm this observation.

\section{Appendix A: Coefficients Occurring in Eqs. (16)}

The coefficients of the aeroelastic governing system represented in state-space form [Eqs. (16)]:

$$
\begin{gathered}
a_{1}^{(3)}=-\frac{\bar{\omega}^{2} r_{\alpha}^{2}}{V^{2}\left(r_{\alpha}^{2}-\chi_{\alpha}^{2}\right)} \\
a_{2}^{(3)}=\frac{M_{\infty} \mu \chi_{\alpha} r_{\alpha}^{2}\left(1+\psi_{1}\right)-V^{2} \gamma\left[\left(x_{0}-1\right) \chi_{\alpha}+r_{\alpha}^{2}\right]}{V^{2} M_{\infty} \mu\left(r_{\alpha}^{2}-\chi_{\alpha}^{2}\right)}
\end{gathered}
$$

$$
\begin{gathered}
\delta_{A} a_{222}^{(3)}=-\frac{M_{\infty}(1+\kappa) \gamma^{3}\left[\left(x_{0}-1\right) \chi_{\alpha}+r_{\alpha}^{2}\right]}{12 \mu\left(r_{\alpha}^{2}-\chi_{\alpha}^{2}\right)} \\
\delta_{S} a_{222}^{(3)}=B \frac{\chi_{\alpha} r_{\alpha}^{2}}{V^{2}\left(r_{\alpha}^{2}-\chi_{\alpha}^{2}\right)} \\
a_{4}^{(3)}=\frac{V \gamma\left[\left(3 x_{0}^{2}-6 x_{0}+4\right) \chi_{\alpha}+3\left(x_{0}-1\right) r_{\alpha}^{2}\right]+6 M_{\infty} \mu \zeta_{\alpha} \chi_{\alpha} r_{\alpha}^{2}}{3 V M_{\infty} \mu\left(r_{\alpha}^{2}-\chi_{\alpha}^{2}\right)} \\
\delta_{C} a_{222}^{(3)}=\psi_{2} \frac{\chi_{\alpha} r_{\alpha}^{2}}{V^{2}\left(r_{\alpha}^{2}-\chi_{\alpha}^{2}\right)} \\
a_{4}^{(4)}=-\frac{\bar{\omega}^{2} \chi_{\alpha}}{V^{2}\left(r_{\alpha}^{2}-\chi_{\alpha}^{2}\right)} \\
a_{2}^{(4)}=\frac{V^{2} \gamma\left(x_{0}-1+\chi_{\alpha}\right)-M_{\infty} \mu r_{\alpha}^{2}\left(1+\psi_{1}\right)}{V^{2} M_{\infty} \mu\left(r_{\alpha}^{2}-\chi_{\alpha}^{2}\right)} \\
a_{3}^{(4)}=\frac{\gamma^{3} M_{\infty}(1+\gamma)\left(x_{0}-1+\chi_{\alpha}\right)}{12 \mu\left(r_{\alpha}^{2}-\chi_{\alpha}^{2}\right)} \\
\delta_{S} a_{222}^{(4)}=-B \frac{r_{\alpha}^{2}}{V^{2}\left(r_{\alpha}^{2}-\chi_{\alpha}^{2}\right)} \\
V_{0}^{(4)}=-\psi_{2}^{2} \frac{r_{\alpha}^{2}}{V x_{\infty}^{2}\left(x_{0}^{2}-\chi_{\alpha}^{2}\right)}
\end{gathered}
$$

Appendix B: Coefficients $A_{k t s}^{(j)}$ Intervening in Eq. (23)

The various elements of Eq. (24), evaluated on the instability boundary, are expressed as

$$
\begin{gathered}
A_{111}^{(1)}=\left(1 / \Delta_{0}\right)\left(\hat{\alpha}_{13} a_{222}^{(3)}+\hat{\alpha}_{14} a_{222}^{(4)}\right) \alpha_{21}^{3} \\
A_{222}^{(2)}=\left(1 / \Delta_{0}\right)\left(\hat{\alpha}_{23} a_{222}^{(3)}+\hat{\alpha}_{24} a_{222}^{(4)}\right) \alpha_{22}^{3} \\
A_{112}^{(2)}=\left(1 / \Delta_{0}\right)\left(\hat{\alpha}_{23} a_{222}^{(3)}+\hat{\alpha}_{24} a_{222}^{(4)}\right) \alpha_{22} \alpha_{12}^{2} \\
A_{122}^{(1)}=\left(1 / \Delta_{0}\right)\left(\hat{\alpha}_{13} a_{222}^{(3)}+\hat{\alpha}_{14} a_{222}^{(4)}\right) \alpha_{12} \alpha_{22}^{2}
\end{gathered}
$$

where the adjoint of a matrix is defined as $\Delta_{0}\left[\alpha_{j p}\right]^{-1}$ and $\Delta_{0}=\left\|\alpha_{i j}\right\|$ is the determinant of the matrix of coefficient $\alpha_{i j}$. In addition, the parameters $\alpha_{i j}$ are

$$
\begin{aligned}
\alpha_{12} & =c\left|\begin{array}{ll}
a_{2}^{(2)} & a_{4}^{(2)} \\
a_{2}^{(1)} & a_{4}^{(1)}
\end{array}\right|+c\left|\begin{array}{ll}
a_{3}^{(3)} & a_{4}^{(3)} \\
a_{3}^{(1)} & a_{4}^{(1)}
\end{array}\right| \\
\alpha_{21} & =\left|\begin{array}{lll}
a_{3}^{(3)} & a_{1}^{(3)} & a_{4}^{(3)} \\
a_{3}^{(1)} & a_{1}^{(1)} & a_{4}^{(1)} \\
a_{3}^{(2)} & a_{1}^{(2)} & a_{4}^{(2)}
\end{array}\right|-c^{2} a_{4}^{(2)} \\
\alpha_{22} & =c\left|\begin{array}{ll}
a_{1}^{(1)} & a_{4}^{(1)} \\
a_{1}^{(2)} & a_{4}^{(2)}
\end{array}\right|+c\left|\begin{array}{ll}
a_{3}^{(3)} & a_{4}^{(3)} \\
a_{3}^{(2)} & a_{4}^{(2)}
\end{array}\right|
\end{aligned}
$$


Appendix C: Coefficients $A_{1}$ and $A_{2}$ Appearing in Eq. (28c)

$$
\begin{aligned}
A_{1}= & \frac{\left(\delta_{S} B+\delta_{C} \psi_{2}\right) r_{\alpha}^{2}}{\left(r_{\alpha}^{2}-\chi_{\alpha}\right)}\left[\left(\Im_{13} \chi_{\alpha}-\Im_{14}\right) \alpha_{21}^{3}+\left(\Im_{23} \chi_{\alpha}-\Im_{24}\right) \alpha_{22}^{3}\right. \\
& \left.+\left(\Im_{23} \chi_{\alpha}-\Im_{24}\right) \alpha_{22} \alpha_{12}^{2}+\left(\Im_{13} \chi_{\alpha}-\Im_{14}\right) \alpha_{12} \alpha_{22}^{2}\right] \\
A_{2}= & \frac{\delta_{A} M_{\infty}(1+\kappa) \gamma^{3}}{12 \mu\left(r_{\alpha}^{2}-\chi_{\alpha}\right)}\left\{\left\{\Im_{13}\left[\left(x_{0}-1\right) \chi_{\alpha}+r_{\alpha}^{2}\right]\right.\right. \\
& \left.-\Im_{14}\left(x_{0}-1+\chi_{\alpha}\right)\right\} \alpha_{21}^{3}+\left\{\Im_{23}\left[\left(x_{0}-1\right) \chi_{\alpha}+r_{\alpha}^{2}\right]\right. \\
& \left.-\Im_{24}\left(x_{0}-1+\chi_{\alpha}\right)\right\} \alpha_{22}^{3}+\left\{\Im_{23}\left[\left(x_{0}-1\right) \chi_{\alpha}+r_{\alpha}^{2}\right]\right. \\
& \left.-\Im_{24}\left(x_{0}-1+\chi_{\alpha}\right)\right\} \alpha_{22} \alpha_{12}^{2}+\left\{\Im_{13}\left[\left(x_{0}-1\right) \chi_{\alpha}+r_{\alpha}^{2}\right]\right. \\
& \left.\left.-\Im_{14}\left(x_{0}-1+\chi_{\alpha}\right)\right\} \alpha_{12} \alpha_{22}^{2}\right\}
\end{aligned}
$$

\section{Acknowledgments}

The support of this research by the NASA Langley Research Center through Grants NAG-1-2281 and NAG-1-01007 is acknowledged. Piergiovanni Marzocca would like also to acknowledge the advice supplied by Gianfranco Chiocchia from the Aeronautical and Space Department, Politecnico di Torino, Turin, Italy. The authors would like to express their gratitude also to the anonymous referees for their constructive comments on the various issues of the paper.

\section{References}

${ }^{1}$ Bolotin, V. V., Nonconservative Problems of the Theory of Elastic Stability, Macmillan, New York, 1963, pp. 274-312.

${ }^{2}$ Librescu, L., "Aeroelastic Stability of Orthotropic Heterogeneous Thin Panels in the Vicinity of the Flutter Critical Boundary," Journal de Mécanique, Pt. 1, Vol. 4, No. 1, 1965, pp. 51-76.

${ }^{3}$ Librescu, L., "Aeroelastic Stability of Orthotropic Heterogeneous Thin Panels in the Vicinity of the Flutter Critical Boundary," Journal de Mécanique, Pt. 2, Vol, 6, No. 1, 1967, pp. 133-152.

${ }^{4}$ Librescu, L., "Aeroelastic Stability of Anisotropic Multilayered Thin Panels," Elastostatics and Kinetics of Anisotropic and Heterogeneous ShellType Structures, Noordhoff International Leyden, The Netherlands, 1975, pp. 106-158.

${ }^{5}$ Dowell, E. H., Aeroelasticity of Plates and Shells, Kluwer Academic, Dordrecht, The Netherlands, 1974, pp. 35-49.

${ }^{6}$ Friedman, P., and Hanin, M., "Supersonic Non Linear Flutter of Orthotropic or Isotropic Panels with Arbitrary Flow Direction,"Israel Journal of Technology, Vol. 6, No. 1-2, 1968, pp. 46-57.

${ }^{7}$ Lee, B. H. K., Price, S. J., and Wong, Y. S., "Non-Linear Aeroelastic Analysis of Airfoils: Bifurcation and Chaos," Progress in Aerospace Sciences, Vol. 35, No. 3, 1999, pp. 205-334.

${ }^{8}$ Lee, I., and Kim, S. H., "Aeroelastic Analysis of a Flexible Control
Surface with Structural Nonlinearity," Journal of Aircrafi, Vol. 32, No. 4, 1995, pp. 868-874.

${ }^{9}$ Mei, C., "Finite Element Approach of Nonlinear Panel Flutter," AlAA Journal, Vol. 15, No. 6, 1977, pp. 1107-1110.

${ }^{10}$ Morino, L., "A Perturbation Method for Treating Nonlinear Panel Flutter Problems," AIAA Journal, Vol. 7, No. 3, 1969, pp. 405-411.

${ }^{11}$ Strganac, T. W., Ko, J., Thompson, D. E., and Kurdila, A. J., "Identification and Control of Limit-Cycle Oscillations in Aeroelastic Systems," Journal of Guidance, Control, and Dynamics, Vol. 23, No. 6, 2000, pp. 1127-1133.

${ }^{12}$ Hopf, E., "Bifurcation of a Periodic Solution from a Stationary Solution of a System of Differential Equations," Berlin Mathematische Physics Klasse, Sächsischen Akademic der Wissenschaften Leipzig, Vol. 94, 1942, pp. 3-32.

${ }^{13}$ Holmes, P. J., "Bifurcations to Divergence and Flutter in Flow-Induced Oscillations: A Finite-Dimensional Analysis," Journal of Sound and Vibration, Vol. 53, No. 4, 1977, pp. 471-503.

${ }^{14}$ Mastroddi, F, and Morino, L., "Limit-Cycle Taming by Non-Linear Control with Application to Flutter," Aeronautical Journal, Vol. 100 No. 999, 1996, pp. 389-396.

${ }^{15}$ Librescu, L., "On the Effect of Physical Nonlinearities in the Aeroelastic Stability Problem of Supersonic Panels," Physical Nonlinearities in Structural Analysis, edited by J. Hult and J. Lemaitre, Springer-Verlag, Berlin, 1981, pp. 156-159.

${ }^{16}$ Breitbach, E. J., "Effects of Structural Nonlinearities on Aircraft Vibration and Flutter," AGARD TR, Vol. 665, 1977.

${ }^{17}$ Chandiramani, N. K., Librescu, L., and Plaut, R., "Flutter of Geometrically Imperfect Shear-Deformable Laminated Flat Panels Using Non-Linear Aerodynamics," Journal of Sound and Vibration, Vol. 192, No. 1, 1996, pp. $79-100$.

${ }^{18}$ Bisplinghoff, R. L., and Ashley, H., Principles of Aeroelasticity, Dover, New York, 1996, pp. 217-234.

${ }^{19}$ Ashley, H., and Zartarian, G., "Piston Theory-A New Aerodynamic Tool for the Aeroelastician," Journal of Aeronautical Science, Vol. 23, No. 10, 1956, pp. 1109-1118.

${ }^{20}$ Lighthill, M. J., "Oscillating Airfoils at High Mach Numbers," Journal of Aeronautical Science, Vol. 20, No. 6, 1953, pp. 402-406.

${ }^{21}$ Carafoli, E., and Berbente, C., "Determination of Pressure and Aerodynamic Characteristics of Delta Wings in Supersonic-Moderate Hypersonic Flow," Revue Roumaine des Sciences Techniques. Serie de Mecanique Appliquee, Vol. 11, No. 3, 1966, pp. 587-613.

${ }_{2}^{2}$ Carafoli, E., Wing Theory in Supersonic Flow, International Series, Monographs in Aeronautics and Astronautics, Vol. 7, Pergamon, 1969, pp. 463-524.

${ }^{23}$ Garrick, I. E., and Rubinow, S. I., "Flutter and Oscillating Air-Force Calculations for an Airfoil in a Two-Dimensional Supersonic Flow," NACA TN-1158 and NACA TR-846, Oct. 1946.

${ }^{24}$ Bautin, N. N., The Behaviour of Dynamical Systems Near the Boundaries of the Domain of Stability, Nauka, Moscow, 2nd ed., 1984, pp. 124-150 (in Russian).

${ }^{25}$ Lyapunov, A. M., General Theory of the Stability of Motion, Moscow, 1950, pp. 1-280 (English translation by Academic Press, New York, 1966).

${ }^{26}$ Malkin, I. G. Theory of Stability of Motion, Translation Series, AECtr-3352, Physics and Mathematics, 1963, pp. 178-184 (translated from Russian, U.S. Atomic Energy Commision, Oak Ridge, TN). 

